LBL --31196

DE92 000810

\title{
Three-Dimensional Simulation of a Hole-Coupled FEL Oscillator
}

\author{
S. Krishnagopal, M. Xie, K.-J. Kim and A. Sessler ${ }^{*}$ \\ Lawrence Berkeley Laboratory, University of California, Berkeley, California 94720
}

August 1991

\footnotetext{
"Work supported by the Director, Office of Energy Research, Office of High Energy and Nuclear Physics, Division of High Energy Physics, of the U.S. Department of Energy under Contract No. DEAC03-76SF00098
} 


\title{
Three-Dimensional Simulation of a Hole-Coupled FEL Oscillator
}

\author{
S.Krishnagopal, M.Xie, K.-J.Kim, A.Sessler \\ Lawrence Berkeley Laboratory, University of California, Berkeley, CA 94720
}

The performance of a two-mirror resonator with holes for out-coupling has been examined in a previous study in which the FEL gain was neglected, but the geometrical effect of the wiggler aperture was included in the optical calculation. The phenomenon of mode degeneracy was found to occur, that has serious implications for the stability of performance when the FEL gain is included. We have developed a FEL oscillator code based on 'TDA', a three-dimensional FEL amplifier code, to study the mode characteristics in the presence of an FEL. We find that the interaction of the radiation and the FEL has an important, and positive, impact on the modeprofile and related cavity performance parameters. In particular, mode degeneracy is not expected to be a serious problem for reasonable FEL oscillator designs. 


\section{INTRODUCTION}

Earlier work [1] has studied the nature of cavity modes in a two-mirror resonator system with a hole at the center of one of the mirrors for coupling out the radiation. It was found that resonators in near-concentric geometry can develop mode degeneracy, which should be avoided for stable operation of a free-electron laser (FEL) in the oscillator mode. However, this study was performed for a cold-cavity, i.e. the effect of the FEL gain was not included. Since the interaction of the FEL and the radiation has the potential to alter the mode-profile significantly, it was decided to extend the previous work by simulating an FEL within the cavity.

We give some details of the simuiation in Section II. Results of the simulation are presented and discussed in Section III. We summarize our conclusions and sketch future directions in Section IV.

\section{NUMERICAL SIMULATION}

We started with the computer simulation code 'TDA', developed by Tran and Wurtele [2]. This is a time-independent, Three-Dimensional, Axisymmetric FEL code. It uses the nonlinear KMR equations [3] of motion for the electrons, and the paraxial wave equation for the radiation. Longitudinal space-charge effects are taken to be negligible (Comptonregime assumption). The code correctly models the single-pass amplification process in an FEL.

To model an FEL oscillator one has to transport the electromagnetic radiation from the end of the wiggler back to its entrance. To implement this we added three segments of a Fresnel-Kirchhoff integral [4] that propagate the radiation (a) from the end of the FEL, to the right-hand mirror (we assume that the electrons travel to the right), (b) from this mirror backwards to the left-hand mirror, and (c) from the left-hand mirror onward to the entrance of the FEL. (In the second transport it is assumed that the radiation 
does not interact with the electron beam and does not see the wiggler aperture.) This completes one pass. The electric field at the entrance of the wiggler is then used as input to TDA, and the process iterated for as many passes as necessary; usually until the mode stabilizes and the optical beam power saturates.

The FEL oscillator code was benchmarked by running without any current in the FEL, thus reducing it to the cold-cavity case, and comparing the mode-profile and cavity loss with the simulations of Ref. 1; the agreement was excellent. Another method of including FEL gain, in the low-gain regime, is to compute an FEL transfer map based on perturbation theory [5]. In the appropriate limit our simulations also agree with this calculation.

In the results presented below, 1024 test particles were used to model the electron beam. The radial grid was discretized into 256 steps. At the outset the radiation was initialized as a Gaussian $T E M_{00}$ mode; on subsequent passes it was, of course, allowed to evolve according to the paraxial wave equation.

At the beginning of each pass a fresh bunch of electrons was loaded. The transverse phase-space coordinates of the test particleo were initialized according to a Hammersley sequence [6]. In the longitudinal dimension, the energies of the test particles had a random Gaussian distribution, while the phases were loaded according to the 'quiet start' scheme [7]. Because of the low initial optical power (relative to the power in the beam), the quiet start scheme was found to be essential for stability and consistency of the results. When, for example, the phases were initialized randomly, even the single-pass gain was found to vary rather widely for different values of the random-number seed. When they were initialized according to a Hammersley sequence, the single-pass gain decreased monotonically with an increase in the prime-number base used to generate the sequence. 


\section{RESULTS}

The simulation code described in the previous section was used to study the influence of the interaction between the radiation and the electron beam, on the mode characteristics and output coupling efficiency of the resonator system. The cavity was near-concentric. The right hand mirror had a hole in the center to out-couple the radiation. The wiggler was placed symmetrically between the two mirrors. The aperture of the wiggler, the wiggler bore, was just the extent of the spatial grid used in TDA. This was not necessarily the same as the cross-sectional radius of the mirrors. Table 1 shows typical parameters used in the simulation.

In order to perform comparisons with the cold-cavity case, we ran the simulation with no current in the FEL. Figure 1 shows a test case in which the dominant cold-cavity mode is the second higher-order mode (two secondary maxima). For the same parameters we then ran with current in the FEL until the total optical power saturated. The dominant mode in this case, also shown in Figure 1, is the fundamental mode. The difference in the two mode-profiles is striking. It is clear that the focussing action of the FEL tends to 'pull' the profile toward the center, resulting in a fundamental mode. It is also remarkable that this action is strong enough to completely bypass the first higher-order mode.

We thus see that when the interaction of the optical radiation with the electrons is taken into account, the dominant mode is not the higher-order mode predicted from cold. cavity simulations. but rather the fundamental. This is an encouraging result for two reasons. Firstly, since the mode is compact within the wiggler, there is greater overlap between the electron and optical beams, and consequently a more efficient extraction of energy from the electrons. This remains true even for larger hole radii. Secondly, the near-Gaussian shape of the fundamental mode allows for better quality and greater stability in the ont-coupled power - an important consideration for design applications.

For the crld-cavity case at small values of the hole radius the domirant mode is 
the fundamental. As the hole radius is increased, the loss into the fundamental increases faster than that into the higher-order mode, until at some point the losses become comparable and one has mode degeneracy. At larger values of the hole radius the higher-order mode becomes the dominant mode. There is therefore a discontinuous change in the mode shape across the region of degeneracy.

Figure 2 shows the hole-coupling efficiency $\eta$ as a function of hole radius. We define the coupling efficiency by $\eta=\alpha_{h} / \alpha_{t}$. Here $\alpha_{h}$ is the ratio of the power radiated through the hole, to the power at the end of the wiggler. For the cold-cavity case $\alpha_{t}$ is the fractional round-trip loss when starting from the end of the wiggler, while for the FEL case it is the fractional power lost in going from the end of the wiggler back to the entrance. Since there is some ambiguity in defining the efficiency for the cold-cavity case , quantitative comparisons of $\eta$ from the figure may not be meaningful. However it is clear that the qualitative behavior is quite different.

For the cold-cavity case $\eta$ rises steadily until one hits the region of degeneracy. Here the fundamental and the higher-order mode are in competition, and the system takes a very long time, hundreds, even thousands, of passes, to reach equilibrium. For reasons of computer time we forbore from taking data in that region. However $\eta$ can be seen to be discontinuous across the region; for larger hole radii $\eta$ starts off at a lower value (than expected by linear extrapolation), before again increasing. With the FEL, on the other hand, $\eta$ is continuous through the region, indicating the absence of mode degeneracy.

This is confirmed by looking at the intensity profiles at points above and below the degeneracy regime. Figure $3(\mathrm{a})$ shows the profiles at a hole radius of $0.9 \mathrm{~mm}$. For both, the cold-cavity and the FEL, cases they are essentially Gaussian, with the latter being perhaps slightly tighter. Even the fact that the FEL does not alter the mode profile at small hole radii (corresponding to small gain and low ioss), is noteworthy.

At a hole radius of $1.6 \mathrm{~mm}$. however, the situation is quite different. The dominant 
cold-carity mode now switches to being a higher-order mode, resulting in the observed discontinuous drop in the hole-coupling efficiency. With FEL gain, however, the dominant mode remains the fundamental. As a result $\eta$ increases continuously, reflecting only the fact that with a bigger hole more of the optical power couples out. Thus, the introduction of the FEL pushes the degeneracy region to much larger values of the hole radius. In fact. up to the maximum value of $2.3 \mathrm{~mm}$ we investigated, there was no sign its onset.

In the design of FEL oscillators it is necessary to avoid mode degeneracy. To date this has been done by using cold-cavity simulations to find the hole radius at which degeneracy sets in, and picking a hole size smaller than that value. Our results indicate, in agreement with the calculations of Ref. 5, that degeneracy actually occurs at larger hole radii than previously calculated; consequently it may be possible to design cavity configurations with bigger holes. This is an important advantage, because it lends greater flexibility in the design of FEL oscillators, allowing for more efficient and broadly tunable hole-coupling schemes.

\section{CONCLUSIONS}

We have developed an FEL oscillator code based on the am, lifier code TI) A developed by Tran and Wurtele. We have used it to study the influence of FEL gain on the modes in a hole-coupled cavity. We find that the interaction of the FEL with the optical radiation plays a benevolent role in the dynamics, resulting in more favorable, nearly Gaussian, mode-profiles. The phenomenon of mode degeneracy, seen in cold-cavity simulations, is not expected to be a problem, at least for a reasonable choice of design parameters.

The above observations have significant implications for the infrared FEL being designed for the (hemical Dymamics Research Laboratory (CIRL) at Lawrence Berkeley Laboratory [8]. We plan to use our oscillator code to explore the optimal cavity design parameters from the viewpoint of improved out-coupling and beam stability. It will also 
be used in an effort to better understand three-dimensional effects in FEL dynamics.

\section{ACKNOWLEDGMENTS}

We wish to thank Jonathan Wurtele for making the code TDA available to us, and Brian Yang for instructing us in its use.

This work was supported by the Director, Office of Energy Research, Office of IIigh Energy and Nuclear Physics, Division of High Energy Physics, of the US Department of Energy under Contract No. DE-AC03-76SF00515. 


\section{REFERENCES}

[1] M.Xie and K.-J.Kim, Nucl. Instr. and Meth. A 304 (1991) 792.

[2] T.M.Tran and J.S.Wurtele, Computer Physics Comm. 54 (1989) 263.

[3] W.M.Kroll, P.L.Morton and M.W.Rosenbluth, IEEE J. Quantum Electron. QE-17 (1981) 1436.

[1] A.G.Fox and T.Li, Bell Syst. Tech. J. 40 (1961) 453.

[5] Performance of a hole-coupled resonator in the presence of FEL gain, M.Xie and K.-J.Kim, these proceedings.

[6] J.M.Hammersley and D.C.Handscomb, Monte Carlo Methods (Methuen, London, 1964).

[7] J.E.LaSala, D.A.C.Deacon and E.T.Scharlemann, Nucl. Instr. and Meth. A 250 (1986) 389.

[S] K.-J.Kim t al., Nucl. Instr. and Meth. A304 (1991) 233. 


\section{FIGURES}

FIG. 1. Mode intensity profiles, for the cold-cavity and with the FEL. In this case the right-and left-hand mirror cross-sections were $15 \mathrm{~mm}$ and $12 \mathrm{~mm}$, respectively.

FIG. 2. Hole-coupling efficiency $\eta$ as a function of the hole radius. With the FEL the rise in $\eta$ is continuous through the region of mode degeneracy. In the cold-cavity case there is a discontinuity. The lines through the data points are drawn only to guide the eye. Both mirrors had a cross-sectional radius of $10 \mathrm{~mm}$.

FIG. 3. Mode-profiles (a) below, and (b) above the region of degeneracy. Both mirrors had a cross-sectional radius of $10 \mathrm{~mm}$. 


\section{TABLES}

TABLE I. Typical parameters used in the simulation

\begin{tabular}{|c|c|}
\hline Parameter & Value \\
\hline Wiggler parameter $\left(a_{w}\right)$ & 0.637 \\
\hline Wiggler length $(L)$ & $3.2 \mathrm{~m}$ \\
\hline Wiggler period $\left(\lambda_{w^{\prime}}\right)$ & $5 \mathrm{~cm}$ \\
\hline Wiggler aperture & $12 \mathrm{~mm}$ \\
\hline Normalized beam emittance $\left(\epsilon_{n}\right)$ & $2 \times 10^{-5} \mathrm{~m}$ \\
\hline Beam radius $\left(r_{b}\right)$ & $0.6 \mathrm{~mm}$ \\
\hline Beam energy $(\gamma)$ & 109.18 \\
\hline Beam current $(I)$ & $100 \mathrm{~A}$ \\
\hline Optical wavelength $(\lambda)$ & $3 \mu \mathrm{m}$ \\
\hline Initial optical power & $10 \mathrm{MW}$ \\
\hline Radius of curvature of mirrors & $4.3 \mathrm{~m}$ \\
\hline Radius of cross-section of mirrors & $10 \mathrm{~mm}$ \\
\hline Separation between the mirrors & $8.2 \mathrm{~m}$ \\
\hline Number of test particles & 1024 \\
\hline Number of radial gird points & 256 \\
\hline Number of longitudinal integration steps & 40 \\
\hline
\end{tabular}




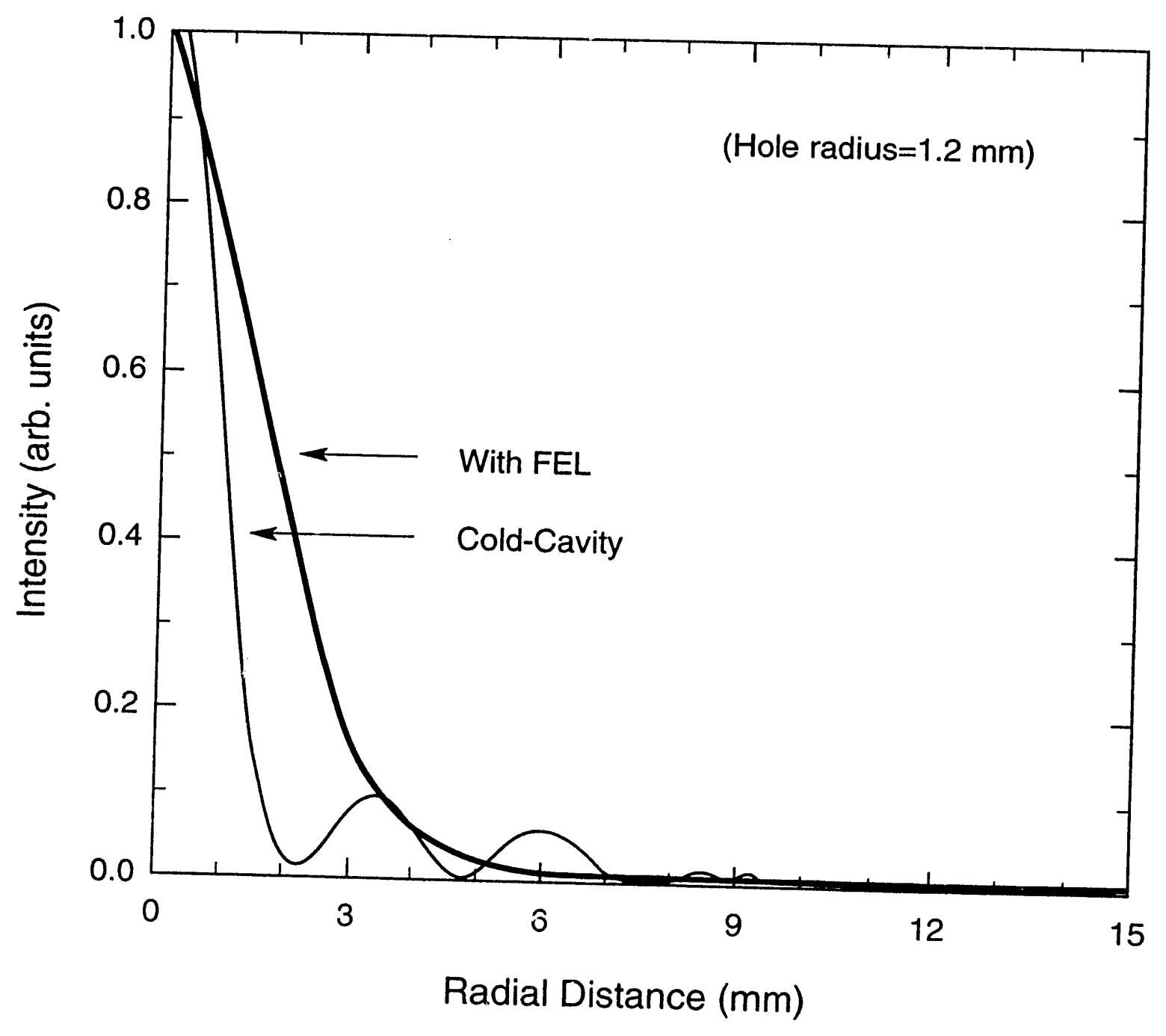




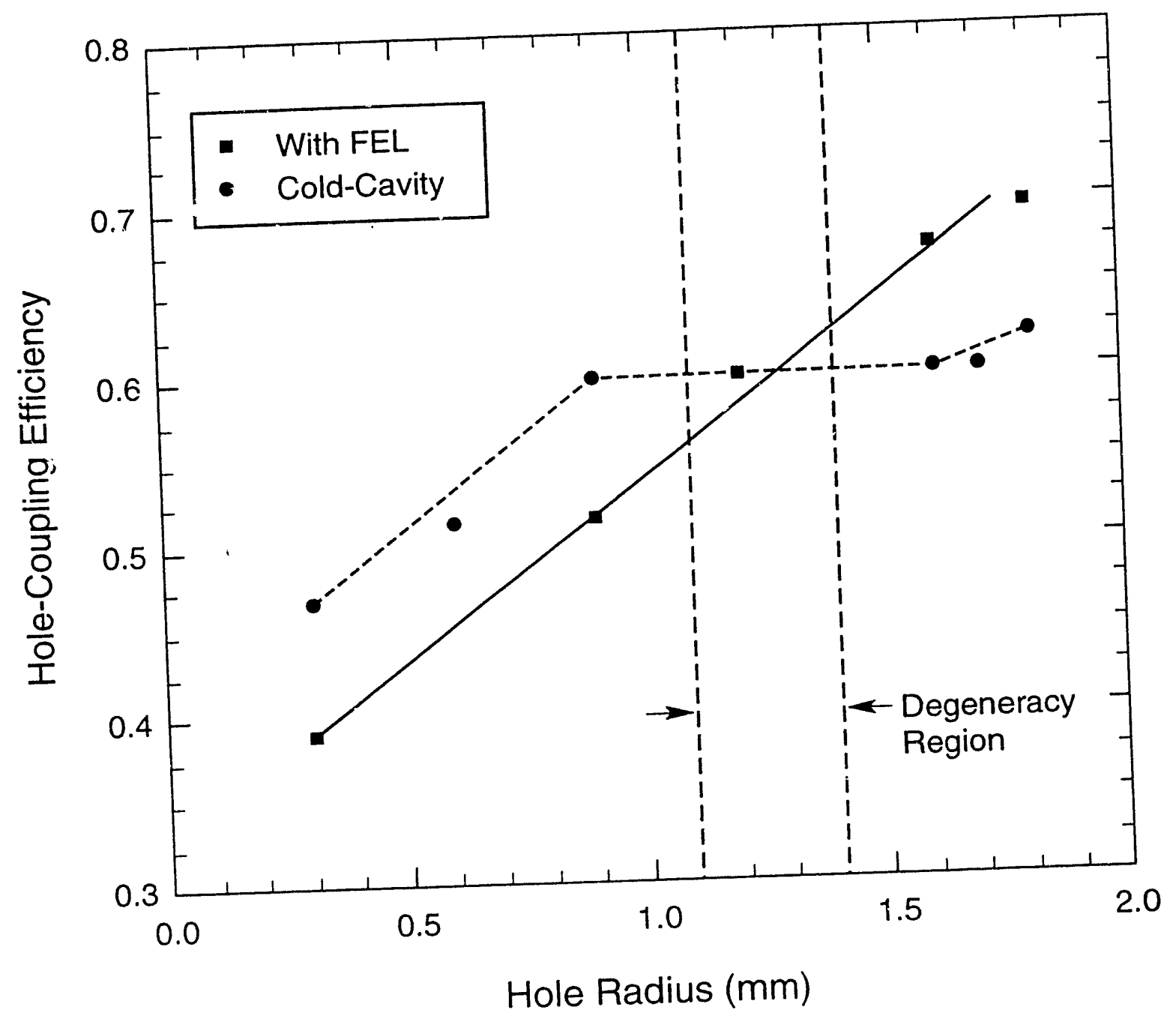




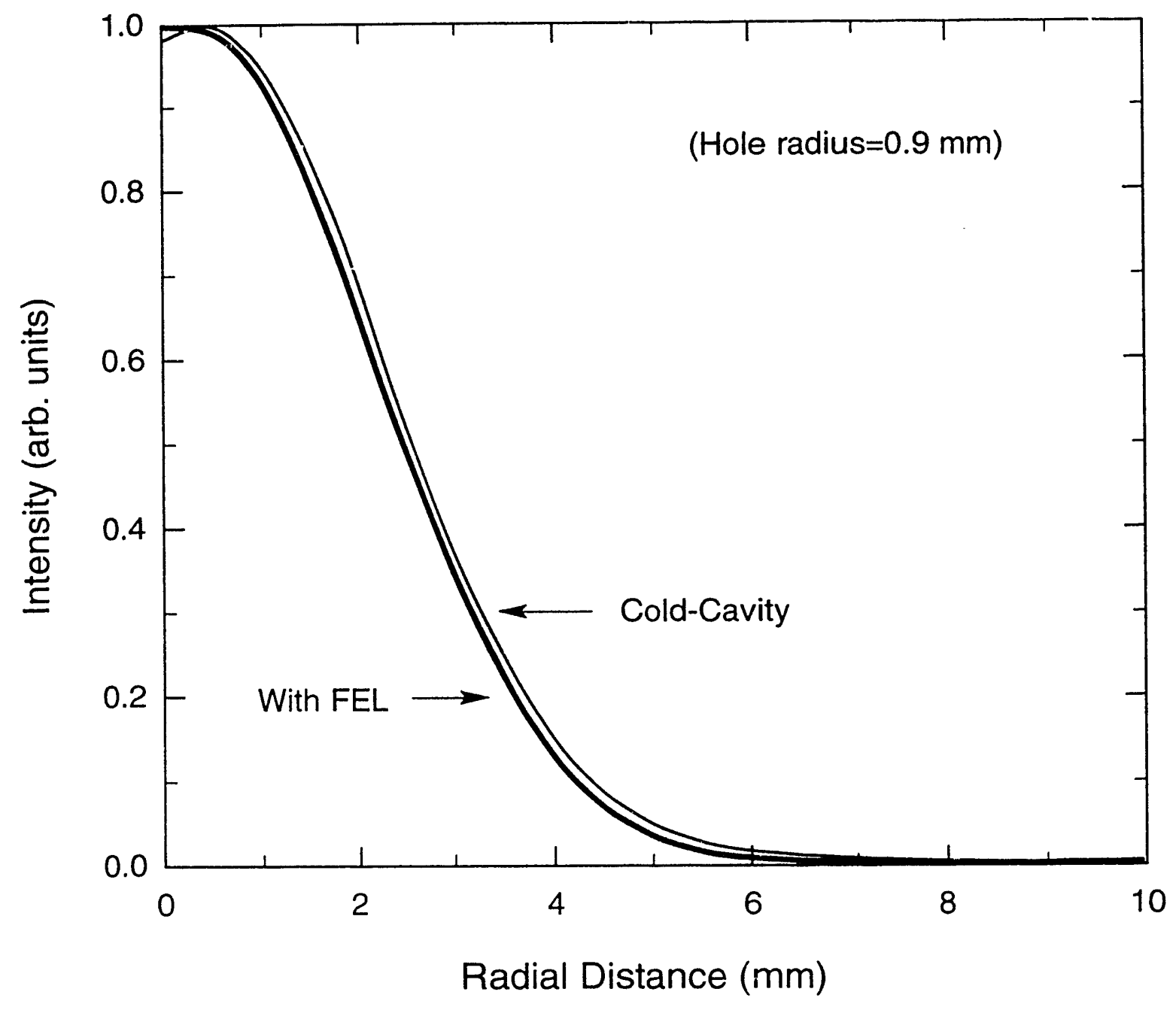




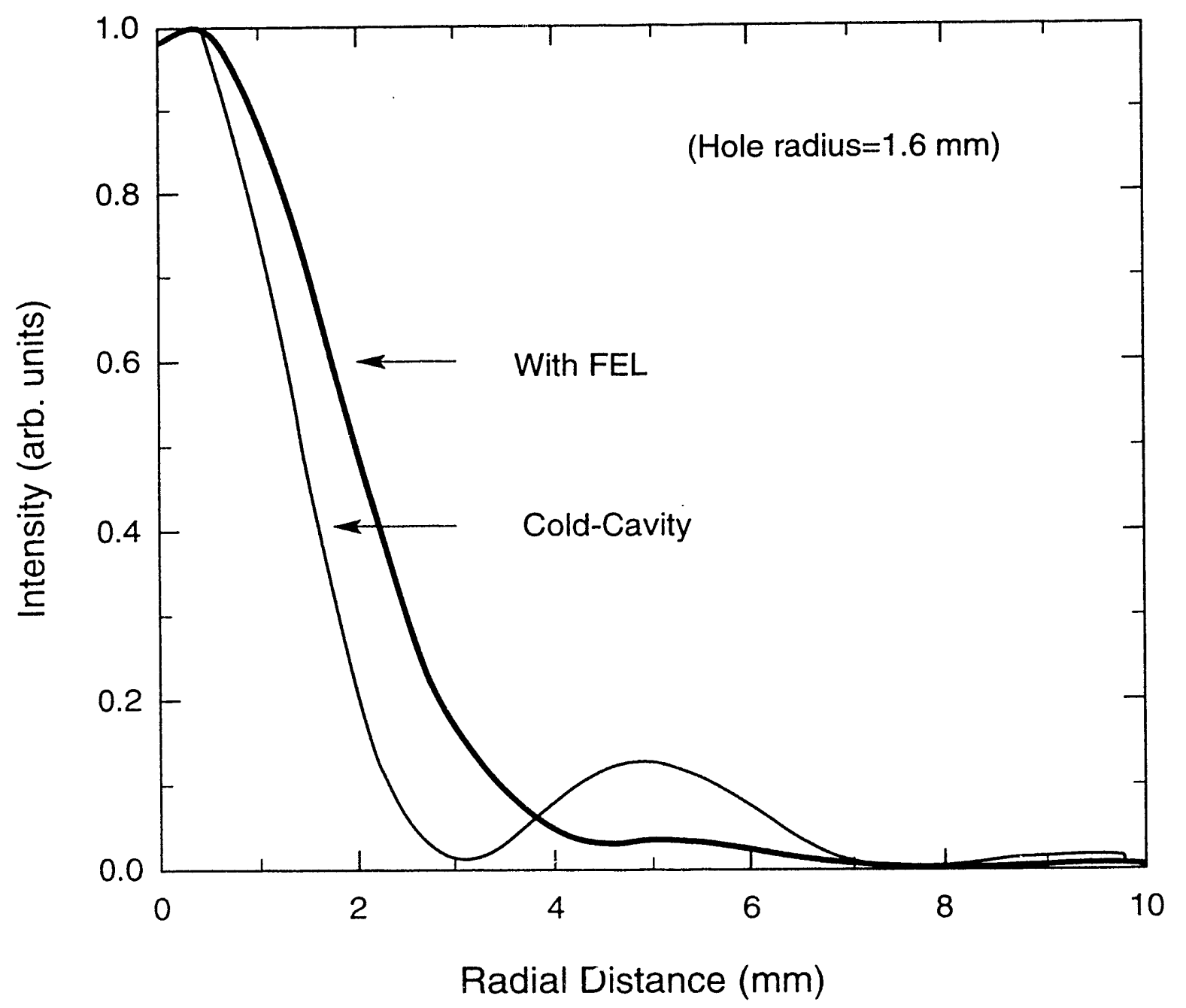



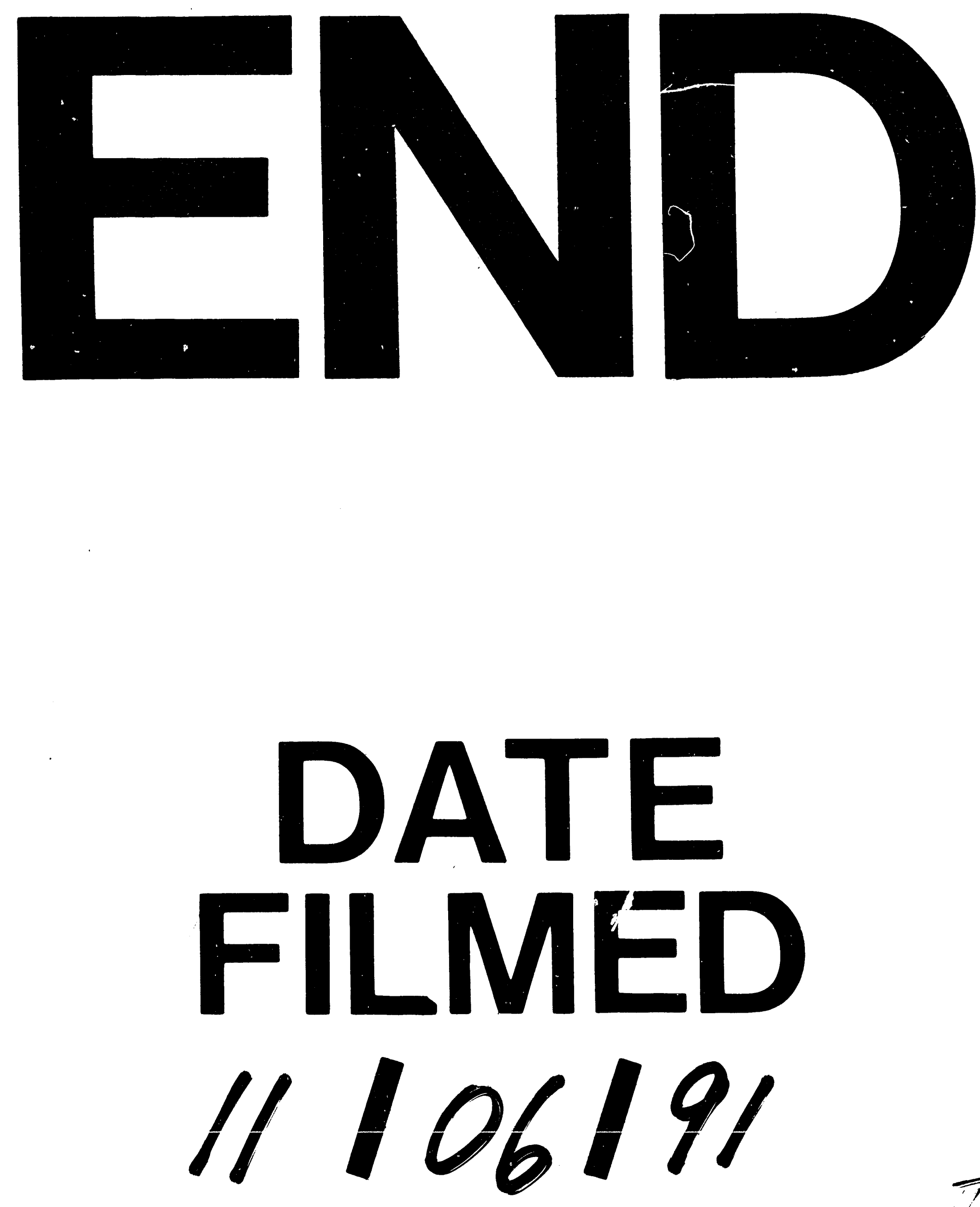
